\title{
Economics of eBay's buyer protection plan
}

\author{
J. Christopher Westland
}

\author{
Correspondence: westland@uic.edu \\ Information and Decision Sciences, \\ University of Illinois - Chicago, 601 \\ S. Morgan Street, UH2400 Chicago, \\ IL, USA
}

\begin{abstract}
Background: This paper explores the financial viability of eBay's Buyer Protection Plan.

Methods: We explore their warranty model assuming exponential auction listing and claims times, under competing assumptions of Normal (classical portfolio theory) versus Paretian claims (industry practice) distributions using a normative algorithm. EBay's model viability was analyzed under three risk metrics - mean-variance risk of classic portfolio theory; value at risk used in regulation such as the Basel Accords; and tail value at risk which is preferred by academics.

Results: Five main findings of the research are:

(1) long-term financial viability is set by boundary conditions that can be managed in eBay's contract terms,

(2) commonly used but simplistic assumptions of Gaussian distributions of contract failures can be misleading, and keeping the Buyer Protection Plan viable demands more realistic distributions,

(3) value at risk measures provide more information about viability than expectations,

(4) tail value at risk measures an lead to sudden and extreme variations in fund value over the parameter range and flawed contracting decisions, and

(5) tail value at risk is the preferable risk measure for assessing Buyer Protection Plan viability, but it needs to be used with an assumption of Lomax contract failures to support profitable contracting.

Conclusions: Our analysis showed substantial improvements in claims management when tail value at risk measures were used instead of conventional VaR measures. This implies that current strategies, and prices for coverage may result in losses to eBay, in current practice.
\end{abstract}

Keywords: Auctions; Insurance; Risk; E-markets; Trust; Reputation

\section{Actuarial structure of eBay's buyer protection plan}

The current analysis assumes that eBay's managerial decision making context involves three decision variables that are stochastic, but partially under the control of the auction market's management reflected in their particular parametric settings.

1. The dispute rate (i.e., the rate at which some sort of transaction failure takes place that is sufficient for the traders to dispute the proper functioning of the market and its transactions. This will be represented by random variable $\tilde{\mathrm{D}}(\mathrm{t}, \mathrm{r})$ which depends on the time of the transaction $t$ and time between failures $r$.

2. The cost to the auction market's owners each time a dispute takes place. This is again a random variable, denoted $\tilde{\mathrm{C}}(m, v, k)$ that depends on three decision 
parameters partially under the control of the market's management: $m$ is the average cost of each dispute; $v$ is the variance of that cost, and $k$ is the maximum cost or cap on payouts that the market is willing to bear. Caps are typical of any sort of indemnification, whether it be warranties, insurance policies, buyer protection or numerous other structures for risk amelioration that typically appear in auction markets.

3. The cost of resolving disputes is a essentially a customer service cost, because incurring it is a proactive managerial decision to increase customer satisfaction by ameliorating problems that result in disputes. Whereas dispute costs are failure costs than are triggered by an event; the ongoing customers service cost is a systems infrastructure cost, and will tend to be a steady payment for maintaining systems. Firms essentially pass this on to their customers (i.e., the traders in a market) through commissions, clearing charges and other fees associated with a given market structure and transaction cost. This 'service systems' charge is equivalent to an insurance premium; it is periodic, non-stochastic, stable over time and represented in this research as $\mathrm{P}(\mathrm{z}, \mathrm{q})$ which depends on $c$ the cost of operating the dispute detection, resolution and correction systems, and $q$ a moral hazard adjustment which reflects the inherent potential of a particular market to suffer abuse - this in turn will depend on the assets being traded, and the control that management places over buyers, sellers, brokers, traders and other players in the market.

The most important parameters will initially be: (1) the failure frequency of auction transactions; (2) the operating and indemnification costs for the buyer protection / insurance program (hereafter called the insurance program) [z]; and (3) the This 'service systems' charge insurance premium $[\mathrm{P}(\mathrm{z}, \mathrm{q})]$ where $z$ the cost of operations mention previously, and $q$ is a cost adjustment depreciation rate (moral hazard declines the longer the insure has paid into the system through subscriptions). These parameters are important in the up-front planning and design of a buyer insurance / protection system. In the longer run, the system needs to allow adjustment of parameters based on claims and operating cost experiences, and these will involve adjustments to other parameters in the model.

When a for-profit online auction company such as eBay creates an insurance program, its objective is to maximize the satisfaction of risk averse customers at a minimum cost for the insurance systems that manages risk. The particular risk that is being controlled arises from information asymmetries: the auction market, e.g., eBay, knows a great deal about the parties on either side of a trade, but these traders don't know much about each other, and don't necessarily trust each other to pay or to deliver a product of expected quality. Assuming that the online auction is a going concern, the problem is a present value maximization problem.

We can summarize the firms problem using minimal notation, where just the main quantities in the problem are expressed. Detailed mathematics with major variables parameterized as functions (either stochastic or scalar) appear in the mathematical appendix at the end of this discussion, along with a table of notation used in the paper. At each time period $t$ the net income from the insurance program will be the premium $P(t)$ less expected warranty claims costs. 
Expected warranty claims will be a product of the number of claims in a time period (random variable $\tilde{C}(t))$ and the amount of each claim $\tilde{D}(t)$. The auction market's problem is to maximize the net present value of this net income over all future time periods

$$
\max (N P V[P-E[\tilde{C} \times \tilde{D}]])
$$

Management's decision parameters are specified in the mathematical appendix, but for this overview, they are not included in the formulas, and the time index is dropped as well. The maximization is subject to a 'safety stock' constraint that dictates that the premium should be high enough to assure that the expected NPV does not decline below a set value $L$ in any single period.

\section{Constraint:}

$$
A-\operatorname{VaR}[\tilde{C} \times E[\tilde{D}]] \leq L
$$

The constraint recognizes that in practice the (stochastic) value of a insurance program will swing between profit and loss. Management wants to assure that premiums, which in online auctions are imbedded in the selling commissions as well as being explicit charges, are sufficiently high so that there are not cash flow problems, or other negative impacts on operations.

The first and second order conditions from the mathematical appendix prove that the maximum value always occurs on the constraint: $A-\operatorname{VaR}[\tilde{C} \times E[\tilde{D}]] \leq L$. In particular, the first order conditions can be simply described for the following parameters:

1. Premiums: optimization on notional premium $P$ is meaningless since you can always increase NPV by raising $P$. This is consistent with the product being viable assuming the initial premium is adequate to cover costs.

2. Costs: optimization on SG\&A cost $c$ implies that dropping SG\&A costs to zero, and discounting all future periods (where claims might be possible) is optimal. Optimization on declining balance rate for SG\&A $q$ are not meaningful - you can always increase NPV by lowering costs. This is consistent with the product being viable assuming the first period premium is adequate to cover costs.

3. Claims (stochastic): optimization for $m, v, k$ involve the payout distribution $F \tilde{C}(x)$ where derivatives for $b_{\max }$ and $b_{c o n}$ are complicated and are explored later in this paper using numerical methods.

4. NPV Discounting: at the margin, the discount rate has declining influence, since $(1+d)^{2}>(1+d)>1$

5. Time to the next dispute: mean time between disputes is $r$ for the exponential distribution, and optimization on $r$ implies that discounting of all future periods, and reducing transaction failures to zero are optimal.

6. Shadow price: this is the marginal change in NPV arising from a change in the constraint fund requirement $\mathrm{L}$ which reflects management's decisions about period-to-period cashflow management for the insurance program.

\section{Structuring a viable online insurance product: the eBay archetype}

This section applies the actuarial modeling developed in the previous section to determine what structures of electronic market transaction insurance would be both 
financial viable, as well as attractive to potential users of the electronic market. Since the behavior of the functions and distributions in the prior section tend to be smooth, without local maxima or minima (indeed, one of the problems in attempting to find optima through Lagrangians) the calculation of fund values using reasonable parameter values can provide a global perspective on the product structures that may be viable, or not.

Internet auction site eBay.com survived the dot-com crash to become the premier, and to a large extent only, US internet auction site (though auctions now constitute less than half of completed sales, the remainder being price-taking transactions). Auction insurance (insofar as they provide coverage as described at http://ebay.com/coverage/) currently provides a baseline for considering the scope and range of coverage that would be attractive to customers. Auction insurance program eBay Buyer Protection protects traders in cases where an item is not received or is not as described in the listing; it excludes Vehicles, Real Estate, Business \& Websites for Sale, classified listings and Business Equipment (replaced by eBay Vehicle Protection Program and eBay Business Equipment Purchase Protection). The ultimate goals are that (1) eBay Buyer Protection be the primary program for settling issues with eBay transactions; (2) there be no double consequences for sellers, and no double refunds for buyers; and (3) chargebacks override all other disputes.

Leading auction site eBay provides their 'Buyer Protection Program' - a full-featured and mature insurance program that is a superset of the warranties provided by other typical auction sites such as uBid, Listia, Webstore and so forth.

Table 1 underscores the dominance of eBay in the U.S. auction market and its significance as an auction firm archetype; it is nearly 1000 times larger than either of the next largest auction sites. The risk management features implemented by eBay reflect the importance of the particular policies implemented in this paper's analysis in the vast majority of online auction transactions taking place in the U.S.

The eBay buyer protection program is essentially an auction transaction insurance policy, where the premium is built into eBay's routine transaction fees. They compensate buyers if they either do not receive an item, or if buyers feel that the item has been misrepresented by the seller (they actually provide a detailed list on their website of situations that would result in possible settlement). Settlements fall into three categories based on the product:

1. Vehicles are covered for the purchase price up to $\$ 50,000$ through eBay Vehicle Purchase Protection

2. Business equipment may be covered for the purchase price up to $\$ 20,000$ through Business Equipment Purchase Protection

3. All other purchases are forwarded to a resolution process that allows for a flexible set of alternatives, most not involving monetary payments by eBay.

Here, insurance takes the form of a performance warranty, and the contract is intended not to look like a life or malpractice insurance (indeed, that is how eBay wants this perceived by its traders). But the entire system essentially sets up an insurance framework. Traders often may not be satisfied with this process, as problem resolution requires time, effort and risk that the settlement will be unsatisfactory. If one of the parties is a criminal (and this is an especially high risk in online auctions because of the anonymity of both parties) then resolution simply may not be possible. 
Table 1 U.S. Online Auctions and their Risk Management Features (Source: http://online-auction-sites.toptenreviews.com/)

\begin{tabular}{|c|c|c|c|c|c|c|c|c|c|c|}
\hline & eBay & Online Auction & eBid & ePier & WebStore & WeBidz & CQout & Auction Fire & Listia & AuctionWarehs \\
\hline Approximate monthly visitors in the U.S. & $120,000,000$ & 39,000 & 36,000 & 13,000 & 124,000 & 839 & $>500$ & $>500$ & 168,000 & $>500$ \\
\hline Auction Listing Fee (monthly) & 50 Free & Free & Free & Free & Free & Free & Free & Free & Free & Free \\
\hline Fixed Price Listing (monthly) & 50 Free & Free & Variable & Free & Free & Free & Free & Free & Free & Free \\
\hline \multicolumn{11}{|l|}{ Auction Fees } \\
\hline Final Value/Closing Fee (per item) & $10 \%$ & Free & $3 \%$ & Free & Free & Free & $3.6 \%-5.29 \%$ & Free & Free & Free \\
\hline Flat Fee (monthly) & na & $\$ 8.00$ & $\$ 7.00$ & na & na & na & na & na & na & na \\
\hline Relisting Fee & Free & Free & Free & Free & Free & Free & Free & Free & Free & Free \\
\hline Product Photos (per listing) & 12 & 4 & 1 & 1 & 4 & 4 & 1 & 1 & 4 & 1 \\
\hline Store Subscription Fees (monthly) & $\$ 15.95-\$ 199.95$ & $\$ 16.00$ & $\$ 99.98$ & $\$ 9.95-\$ 21.95$ & na & $\$ 2.10-\$ 99.95$ & $\$ 5.50-\$ 15.00$ & na & na & na \\
\hline Listing Designer (per listing) & $\$ 0.10-\$ 0.30$ & & & & & & & & & \\
\hline Bold (per listing) & $\$ 2.00-\$ 4.00$ & & $\$ 0.07$ & $\$ 0.50$ & & $\$ 0.01$ & & & & $\$ 0.05$ \\
\hline \multicolumn{11}{|c|}{ Transaction Risk Management Features $1=$ included; $0=$ absent) } \\
\hline Resolution Center & 1 & 0 & 1 & 0 & 0 & 1 & 1 & 0 & 0 & 0 \\
\hline Feedback Options & 1 & 1 & 1 & 1 & 1 & 1 & 1 & 0 & 1 & 1 \\
\hline Buyer Protection Policy & 1 & 0 & 0 & 0 & 0 & 0 & 1 & 0 & 1 & 0 \\
\hline Community Forum & 1 & 1 & 1 & 1 & 1 & 1 & 1 & 1 & 0 & 0 \\
\hline
\end{tabular}


This suggests the desirability of a more explicit 'insurance' policy - where either buyer or seller (or both) buys a policy on a transaction that pays nothing if the transaction is satisfactory (eBay profits) but pays a specific schedule (possibly capped) of amounts for each problem or fault in the transaction.

The eBay "Buyer Protection" insurance schemes are quite simple, and presented to traders more as warranties than as insurance policies. To gain insight into how such a product might develop into a family of cost-effective insurance contracts, we can draw insights from the currently most developed commercial auction insurance industry -automobile auction "payment and title guaranty insurance." Such contracts protect auctions against bad checks and failure to collect from buying dealers; additionally they protect against losses resulting form auctioning of stolen vehicles or other situations in which there are existing claims to title. Automobile dealer online auction structure in the US and Japan have been extensively researched in (Lee et al. 2003; Lee et al. 1999). Such dealer actions are distinguished from consumer $\mathrm{C} 2 \mathrm{C}$ auctions in three respects: (1) traders are dealers that are familiar with the auction and bidding structure, product quality, and moral hazards inherent in each trade; (2) there exists a liquid secondary market for products purchased in error, and often the buyer does not even have to take delivery of the vehicle before putting it again up for sale; and (3) buyers and sellers have comparatively homogeneous beliefs and behavior (compared to a $\mathrm{C} 2 \mathrm{C}$ auction such as eBay) because the treat each trade from a standpoint of return on investment. $\mathrm{C} 2 \mathrm{C}$ traders, in contrast, are more interested in acquiring a rare or cheap item, or selling unused items, than in a micromanaged investment in inventory. Because of these features, automotive auctions have generally found it easier to structure insurance and risk management programs for their markets. Heterogeneous priorities and behaviors in the $\mathrm{C} 2 \mathrm{C}$ market, combined with highly varying degrees of trader sophistication, and lack of a liquid resale market in $\mathrm{C} 2 \mathrm{C}$ auctions make risk management more challenging. Auction markets such as eBay have developed relatively robust risk management approaches that are able to operate in their relatively information poor $\mathrm{C} 2 \mathrm{C}$ environment.

On the auto auction insurance buyer's side, the price of insurance is typically adjustable (in a way that eBay's Buyer Protection is not):

1. to allow risk sharing between the auction and buyer via copayments and deductibles, and

2. to allow pooling of risk using particular pools of funds

In short, eBay insurance claims payout will (approximately) equal the sales price.

EBay's Square Trade warranties start at 45 days after purchase, and can be 1 to 4 years. For sake of the current analysis, assume that coverage lasts for 320 days $=365$ days -45 days. This would imply a window of 320 days for our analysis; therefore:

1. Sales rate $=50$ listing in a320 period $\cong 320 \times 0.157232704$

2. Claims rate $=30$ claims in a320 period $\cong 320 \times 0.09045597$

The previous analysis with Lagrangians provides some insight into the general economics of an online auction insurance product, but these are insufficient to actually define a marketable and profitable product. 
Further insights must be gained through exploration of parameter values. A review of available literature allows me to restrict parameter values. Research (Anythingresearch. com, 2013) cited the following statistics for online auctions:

1. Average final sales price: $\$ 85.95$ (standard deviation: $\$ 91.26$ )

2. Average auction completion rate: $57.53 \%$

3. Average frequency of listings per site member: One listing every 6.36 days

These statistics help us to set the context for offering a generic online auction insurance product.

\section{Risk metrics and claims distributions}

In the context of Classic Portfolio Theory (Rockafellar and Uryasev, 2002; Sharpe and Sharpe, 1970) assumptions of Normal risk and return, such an approximation must assume a bounded variance. A Lomax distribution needs not make such assumptions, and is more suitable for risk and claim distributions (where it is commonly employed). This amount will be used as a representative claim amount in guiding our construction of comparative Normal and Lomax examples.

The minimum reinsurance fund value $\mathrm{L}$ is computed at $10 \%$ of the claims cap $\mathrm{k}$, which provides a reasonable estimate based on experience from other types of casualty insurance (e.g., vehicle insurance). The shadow price $\lambda$ computed for the reinsurance fund amount can be used to assess the robustness of this assumption. The declining balance rate mimics the declining commission payments on most insurance products, and assumes that these are reflected in other selling, general and administrative expenses (S,G \& A) for the product. The transaction time to failure is based on Media Metrix Core Reports statistics.

The actuarial viability of each of these options will be examined in the following sections. Additionally, the auctioneer (e.g., eBay) may be interested in profitability of operations - for example, they might be most concerned with the annuity collections net of costs, with a goal of assuring that the annuity fund is maximized or stays within certain bounds. They would like to assure that anticipated claims payments do not bankrupt the fund; and they may also want assurances that the fund does not exceed a certain value. These requirements are presumed to be idiosyncrasies of particular firms or investment decisions, and are not relevant to the overall viability of insurance products - but in practice they matter to shareholders, and will influence how a firm structures its products.

It is standard in financial portfolio theory to assume that risk is measured by the variance of a Normally distributed random variable. Such assumptions are fundamental to the capital asset pricing model and other financial benchmarks. The insurance industry, in contrast, commonly assumes a Pareto distribution for claims (the Lomax distribution is a Pareto type II distribution with a $[0, \infty)$ support). For the purposes of the following analysis, the Normal $\left(\mu, \sigma^{2}\right)$ and the Lomax $(\alpha, \beta)$ both can be set to have identical means of $\mu$ dollars (e.g., \$85.95 in the case of eBay's average sales value); the Normal density is unimodal, whereas the Lomax has its mode at zero, and is downward sloping for increasing losses. Normal distributions are invoked in Classical Portfolio Theory to describe risk $\sigma^{2}$ and return $\mu$ behavior through models such as the Capital Asset Pricing Model. The insurance industry, in contrast, tends to model wealth, income, payouts and so forth via Pareto distributions. The Lomax distribution is a Pareto type II distribution with its mode at zero. The two distributions are dramatically different: the 
Lomax is defined on a $(0, \infty)$ support, while the Normal is unimodal and defined on $(-\infty, \infty)$. But assuming a risk $\sigma^{2}$ - return $\mu$ context, we can solve for $(\alpha, \beta)$ in terms of $\left(\mu, \sigma^{2}\right)$ to define Normal-Lomax pairs with equal means and variances:

$$
\begin{aligned}
& \alpha=\frac{2 \sigma^{2}}{-\mu^{2}+\sigma^{2}} \\
& \beta=\frac{-\mu^{3}-\mu \sigma^{2}}{\mu^{2}-\sigma^{2}}
\end{aligned}
$$

We will look at these pairs in terms of their performance under various risk and performance objectives for the insurance fund.

Measures of risk have evolved considerably since the classic portfolio theory meanvariance framework. Value-at-Risk (VaR) metrics for fixing the reserves needed to balance (Daníelsson et al., 2001; Moscadelli, 2004) a risky investment portfolio have been important to industry since at least 1996 when the Basel Committee proposed to use $\mathrm{VaR}$ (the quantile of the profit and loss distribution) to determine capital reserves. Since then, a great deal of effort has been devoted to the applications of VaR in determining capital reserves, in portfolio management, and in hedging (for overview, see (Duffie and Pan, 1997, 2001; Gourieroux and Jasiak, 2010). VaR is easy to understand by practitioners, and is legislated into its key position as a metric of risk. Otherwise, it has many undesirable properties, and has been criticized as a facilitator of financial crises such as that in 2008.

Additionally, tVaR has been recommended as a standard measure for calculating insurance fund capital requirement; in 2002, the Life Capital subcommittee of the American Academy of Actuaries suggested the use of $\mathrm{tVaR}$ to set the risk-based capital requirement. (Boyle et al. 2005) show that under a VaR constraint, a trader has the incentive to hold a riskier portfolio that can be subject to huge losses with small probability. (Basak and Shapiro, 2001) study portfolio selection under a VaR constraint and find that an agent tends to invest more in the risky asset than he/she would in the absence of this constraint. Furthermore, VaR is not convex in general, so that an investor may be better off investing in the individual asset separately. Informative risk measures need to account for both the probability of a bad event and also its magnitude. (Artzner et al. 1999) follow a systematic approach and define "coherent risk measures." A risk measure is coherent if it satisfies axioms such as monotonicity, invariance with respect to drift, homogeneity, and subadditivity (VaR is not coherent and violates the subadditivity for most distributions). Tail value at risk (tVaR) takes into account not only the probability of loss but also the magnitude of the loss when a loss occurs. The tVaR metric has been used to derive the efficient portfolio frontier (Rockafellar and Uryasev, 2000, 2002), (Bassett et al. 2004) as in the mean-variance framework to calculate required capital ((John and Hancock 2005) and to perform a sensitivity analysis of portfolio risk (Tasche, 2002),(Fermanian and Scaillet, 2005).

$\mathrm{VaR}$ is not a coherent risk measure because it violates the subadditivity property - i.e., it violates $\operatorname{VaR}(X+Y) \leq \operatorname{VaR}(X)+\operatorname{VaR}(Y)$. In the current paper's context, the risk of losses exceeding reserves in two consecutive periods (or alternatively, for multiple policies) could exceed the sum of the risks in each period (or policies). Surprises such as this lay at the root of many of the underwriting missteps in the financial crash of 2008. $T \operatorname{VaR}_{w}(X)$ calculation 
is slightly more complicated, and can be stated as a function of $\operatorname{VaR}_{w}(X)$ for major actuarial distributions. Table 2 summarizes the functional relationship of VaR to tVaR.(Daníelsson et al., 2001).

Table 2 sets the various parameter values for the analysis of product viability. We will investigate the sensitivity of changes to this base claims rate in our analysis by manipulating the variance $\sigma^{2}$ and failure $\varpi$ of online auction transactions.

Assuming that the operations are stable over time, the NPV discounting simply reduces to a scaling factor $\sum_{t=1}^{n}(1+d)^{-t}$ and we can simplify the risk and profitability analysis to looking at just one time interval, chosen as 320 days in this analysis. The fund balance analysis looks at expectation as well as extreme risks, graphing $O$ $[\tilde{S} \times P-\tilde{C} \times \tilde{D}]$ where $O[\cdot]$ is one of the three operators $\{E(\cdot), \operatorname{VaR}(\cdot), \operatorname{tVaR}(\cdot)\}$ in order to simplify the discussion and analysis.

\section{Insurance fund "at risk" mappings with differing claim and risk assumptions}

This section looks at the behavior online auction insurance through an example that maps the fund value from the previously articulated insurance model assuming eBay's real-world values for pricing and payout, and using various assumptions found in the literature for claims and risk behavior. Specifically:

1. Claim distribution: (1) Normal, (2) Lomax

2. Risk metric: (1) expected loss, (2) value at risk $\mathrm{VaR}_{\gamma}$ and (3) tail value at risk $t V a R_{\gamma}$

3. 'Failures' causing a claim (proportional to listings)

These mappings can provide insight into the behavior of online auction insurance that we would expect to encounter in practice and are summarized in Figs. 1, 2 and 3 for expected loss, value at risk $V a R_{r}$ and tail value at risk $t V a R_{\gamma}$ respectively.

Figure 1 inspects the potential to consider median rather than mean risk, which only makes a difference when assuming Lomax claims. In this case, across parameter settings the effect is small, and generally results in lowered profitability. The graphs for a particular expected claims loss over the range of frequency of transaction failures $\varpi$ and risk levels $\sigma$ with Normal claims distribution is predictable and well behaved. When we shift to a more realistic Lomax distribution, the fund value declines precipitously when the failure exceeds $20 \%$ of transactions.

The fund value changes much more predictably and slowly if instead of expected fund value we measure median fund value. This may be relevant to the debate, since the Normal-mean-variance portfolio framework was developed in a time before computers were widely applied to portfolio analysis, and in such a framework, there is no difference between median and expected values for a Normal claims experience. But differences do arise for other distributions, in particular the Lomax. In a practical

Table 2 Functional Relationship of $V a R$ and $t V a R$

\begin{tabular}{lllll}
\hline Distribution & Uniform $(a, b)$ & $\operatorname{Gamma}(1, b)$ & $\operatorname{Lomax}(a, b)$ & Normal $(m, v)$ \\
\hline$\frac{\operatorname{tVar}(w)}{\operatorname{VaR}(w)}$ & $\frac{b(2-w)+a w}{2(b(1-w)+a w)}$ & $1-\frac{1}{\log (w)}$ & $\frac{a}{a-1} ; a>1$ & $m+\sqrt{v} \frac{f \tilde{c}[\operatorname{VaR}(1-w)]}{1-w}$ \\
$f(w): t \operatorname{VaR}[w]=\operatorname{VaR}[f(w)]$ & $\frac{w}{2}$ & $\frac{w}{e}$ & $w\left(\frac{a}{a-1}\right)^{a}$ & $m+\sqrt{v}(1-f \tilde{c}[\operatorname{VaR}(1-w)])$ \\
\hline
\end{tabular}




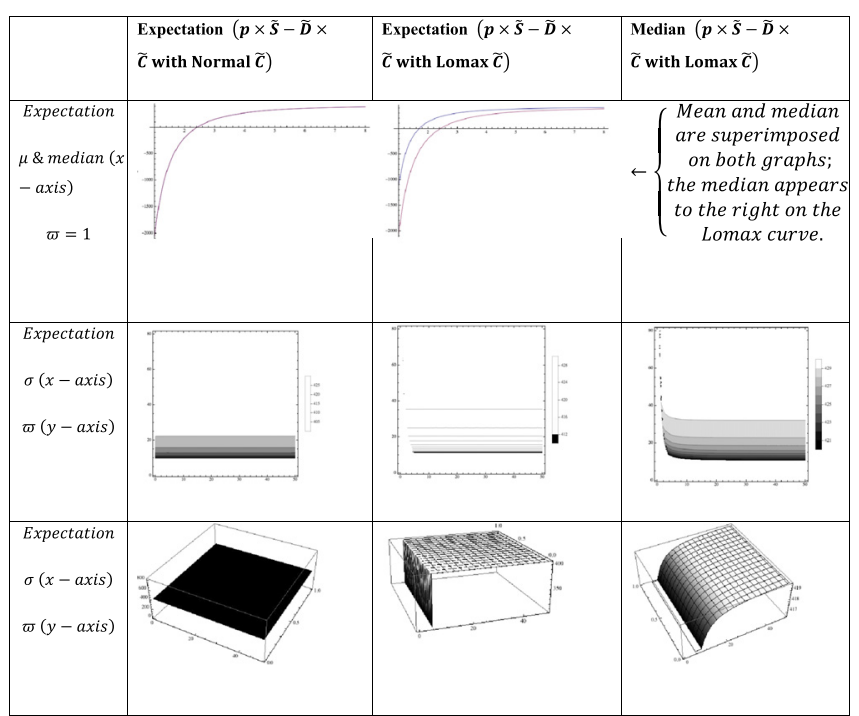

Fig. 1 Fund value with expectation risk metric (with median claim for reference)

situation, the less dramatic behavior of a median loss metric would ease the potential for significant errors in underwriting.

VaR metrics for assessing fund and portfolio values have been important to industry since at least 1996 when the Basel Committee proposed to use VaR (the quantile of the profit and loss distribution) to determine capital reserves. In general, they provide a more meaningful measure of risk, since they describe behavior at the extremes rather than the mean. Figure 2 presents the behavior of the auction insurance fund where VaR measures the risk. In this situation, an additional parameter $\gamma$ the quantile becomes important with fund values changing very quickly at particular 'cutoff' values. This suggests the possibility of catastrophic failures in markets under particular market conditions.

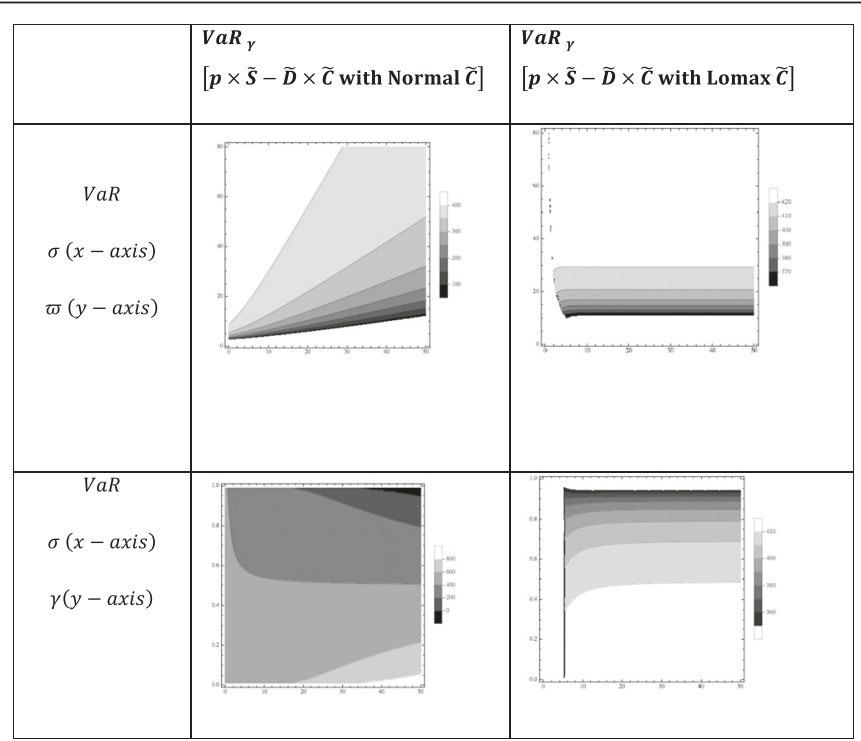

Fig. 2 Fund Value with VaR Risk metric 


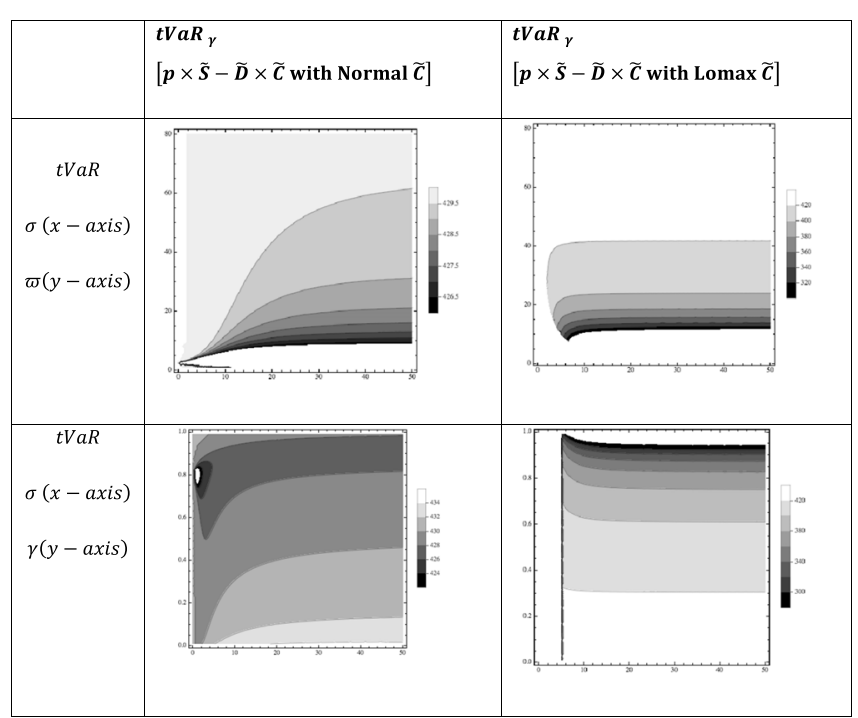

Fig. 3 Fund Valuation with tVaR risk metric

The graphs in Fig. 2 of fund value versus failures show similar behavior for both Normal and Lomax claims distributions. On the other hand, Normal and Lomax results differed substantially as the value at risk quantile varied, and these (as expected) are influenced by the dispersion of the claims distribution. The results for the Normal distribution tend to vary significantly with $\gamma$ and suggests that such models would be less predictable in practice.

In recent years, tVaR has grown increasingly popular in risk assessment (Fig. 3); it takes into account not only the probability of loss but also the magnitude of the loss when a loss occurs, and is a coherent risk measure. Figure 3 shows that an assumption of Normal claims becomes very problematic when $\mathrm{tVaR}$ is used as a risk metric. When the failures approaches about 40 \% singularities appear. This does not happen when Lomax distributions are used to model claims. In a practical setting, if underwriting decisions were made based on Normal claims assumptions, it would be hard to justify any policy when the failures exceed around $10 \%$, even though such policies under a more likely Lomax claims distribution would be profitable. The steepness of the decline in fund value under assumptions of Normal claims implies that simply raising the premium on a policy is not an option - the increases needed would be prohibitive, and likely push demand too much to make the product feasible.

Why would making Normal assumptions lead us to such extremely misleading decisions in the case of online auction insurance underwriting? We can understand this by invoking the Fisher-Tippett-Gnedenko theorem for extreme (tail) values of a distribution. The theorem states that the maximum of a sample of independent, identically distributed random variables after proper renormalization converges in distribution to one of three possible distributions: the Gumbel distribution, the Fréchet distribution, or the Weibull distribution. In the specific examples in this study, the exponential tails of the Exponential and Normal distributions converge to a Gumbel limit; but the fat-tailed Lomax distribution converges to the Fréchet limit (Wolpert, 2012). The tails (and thus tVaRs) of the Normal and Lomax distributions are at a fundamental level, substantially different in structure. There is considerably more weight in a Fréchet tail (and this 
implies that there will generally be much less weight in the central body of the Lomax distribution and more $t \mathrm{VaR}_{\gamma}$ at a given $\gamma$ ). With the Normal distribution, we expect the opposite effect - a higher frequency of middling claims.

A look at the periodic fund increment $p \times \tilde{S}-\tilde{D} \times \tilde{C}$ provides additional insight. The random variable for auction listings $\tilde{S}$ and claims frequency $\tilde{D}$ are both exponentially distributed, and at the extreme converge to the same Gumbel limit as a Normal $\tilde{C}$. This makes them sensitive to changes in distribution parameters, since at the extreme, all three variables are moving together, and $\tilde{D} \times \tilde{C}$ is likely responsible for volatility appearing in fund values in this simulation based on eBay's parameters. In contrast, if claims $\tilde{C}$ are Lomax, then the tails for listings $\tilde{S}$ are Gumbel, while the tails of $\tilde{C}$ are the fatter Fréchet. There may be a smaller range of parameters, under the Lomax claims assumption, to keep the fund value positive, but the transition to a fund deficit will be more gradual, as is reflected in the graphs of the fund surfaces.

\section{Conclusions and discussion}

The first order conditions for optimizing an online auction insurance contract prove that an optimal online auction insurance contract may be constructed from boundary conditions over which management of the underwriting firm has control, rather than interior solutions which are dependent on the various distributions of random variables influencing the insurance. This is significant, since it implies that the results of this research can be adopted and used by management in their decision making.

The specific design and managerial parameters which insurance providers have at their disposal include:

1. Premiums consistent with the product being viable assuming the initial premium are adequate to cover costs.

2. Costs: consistent with the product being viable assuming the first period premium is adequate to cover costs.

3. Payouts under claims are complicated and are explored later in this paper in the example

4. NPV Discounting at the margin, the discount rate has declining influence, since $(1+d)^{2}>(1+d)>1$.

5. Less frequent failures are desirable, and reducing the Internet transaction failures to zero are optimal.

6. Shadow price is computed as a byproduct of the model building, and may be considered the cost of setting a particular fund constraint L. As the shadow price as a function of L is likely to be highly non-linear, knowing the shadow price can help design more manageable and profitable strategies for buyer protection insurance programs.

The potential for applications by insurance underwriters was explored through an example with parameters based on the internet auction site eBay.com which offers auction insurance program eBay Buyer Protection protects traders in cases where an item is not received or is not as described in the listing; it excludes Vehicles, Real Estate, Business \& Websites for Sale, classified listings and Business Equipment (replaced by eBay Vehicle Protection Program and eBay Business Equipment Purchase Protection). 
It should be noted that the insights made available by this research have been due largely to the ability to should that optimization yields solutions on the constraints (because any interior solutions lie in the complex plane). Global optima can, and often are at solutions where the derivatives, with respect to the one particular variable that is what the objective function is optimized on, are not zero. We might for example consider a hyperplane that has a 45 degree slope with respect to every axis. If we optimize under the constraints $\left\{x_{i} \in[0\right.$, 1] $\forall i\}$ then the maximum is at the point $\left\{x_{i}=1 \forall i\right\}$ and minimum at $\left\{x_{i} \in 0 \forall i\right\}$ and at both the slope is 45 degrees. These are boundary solutions, and they are common in optimization. If this were our business problem, we would only have to look at one point (depending on whether it is minimization or maximization). Additionally it should be noted that multivariate optimization does not mean multi-objective optimization. In particular, if you are looking for an interior solution (where the gradient is level) there is only one objective function. But business problems generally only have one objective function - long-run profit, which for electronic markets is long run time discounted profitability, or net present value of the fund. Multi-objective optimization is generally more useful for social welfare problems, where competing interests set multiple objectives, and Core Theory may yield non-unique and inherently ambiguous decisions. The solutions to optimal e-commerce transaction insurance problem lie on the constraints, and thus a constraintby-constraint inspection of required parameters for optimization is sufficient to provide assurances that we have a viable business model.

For management decisions to be viable, they must assure that the future value of the insurance fund is positive, or at least does not run a deficit. The prediction statistics are involved, and there are various metrics promoted in the academic literature to measure viability. Specifically we explored three risk metrics: (1) expected loss, (2) value at risk and (3) tail value at risk. Expected loss is widely invoked in academic finance literature, but leaves an insurance company exposed to extreme 'Black Swan' losses. Finance and insurance regulation instead enforce using a value at risk (VaR) metric, which unfortunately is not coherent and violates the subadditivity property - i.e., it violates $\operatorname{VaR}(X+Y) \leq \operatorname{VaR}(X)+\operatorname{VaR}$ $(Y)$ - for most distributions. The tail value at risk ( $\mathrm{tVaR})$ measure takes into account not only the probability of loss but also the magnitude of the loss when a loss occurs and is preferred over the other two measures by many academics. It would be even more popular were it not for the computational complexity it lends to risk assessment.

The insurance model constructed in the first part of the paper was tested assuming a Normal loss (consistent with classic portfolio theory) and Lomax claims loss as is used in most insurance work. The insurance model set parameters based on our best knowledge of eBay's real-world values for pricing and payout, and using various assumptions found in the literature for claims and risk behavior. With these parameters, several striking results were derived from the example.

Fund behavior assuming a Normal claims distribution is predictable and well behaved, but when we shift to a more realistic Lomax distribution, the fund value declines precipitously when the failures exceeds $20 \%$. In a practical situation, the less dramatic behavior of a median loss metric would ease the potential for significant errors in underwriting.

When the expected loss risk metric is replaced with a VaR metric for assessing fund and portfolio values, both Normal and Lomax claim distributions provide somewhat similar results. Increasing failure dispersion of the payout distribution lead to higher losses.

Replacing the VaR metric with the preferable tVaR metric for assessing fund and portfolio values leads to more interesting conclusions. Under $\mathrm{tVaR}$, Normal claim distributions 
result in erratic fund performance, and it is difficult to divine the cause for changes in fund performance. In particular Normal claims becomes very problematic as failure approaches $40 \%$ with the appearance of abrupt changes in the fund value surface. The practical implications of these abrupt changes is that at these parameter values, an insurance program will be unmanageable. Results under assumption of Lomax claims assumption are, in contrast, regular and thus likely to support more trustworthy underwriting. These differences are explained through the Fisher-Tippett-Gnedenko theorem for extreme (tail) values of the Lomax, Exponential and Normal distributions. We concluded that the use of a tVaR risk metric is not compatible with an assumption of Normal claims payout.

In a practical setting, if underwriting decisions were made based on Normal claims assumptions, it would be hard to justify any policy when failures are more than $10 \%$, even though such policies under a more likely Lomax claims distribution would be profitable. The steepness of this abrupt drop in fund values with Normal claims implies that simply raising the premium on a policy is not an option - the increases needed would be prohibitive, and likely push demand too much to make the product feasible.

The example in this research was constrained by the use of secondary sources for auction data. It proposed a particular Clayton copula for incorporation of primary data into this insurance model. This is an area for future research, and can further refine the applicability of this research.

In markets as diverse and innovative as those in the US, we might expect products similar to the Internet retail insurance product specified above to exist already if they were feasible. But actuarial calculations show that the design of a Internet retail insurance policy is more complex than those embraced in other major insurance sectors (with perhaps the exception of medical malpractice, which is the fifth largest insurance business in the US). The design must balance affordable premiums for what many buyers may see as a discretionary cost with what, until recently, were the poorly understood demographics of Internet retail (where an understanding is needed for policy underwriting), and challenges of designing robust premium and payout schedules to maintain viability of the insurance fund.

\section{Mathematical appendix}

Three variables define the product, whose values are in turn determined by seven parameters affecting the policy of the insured transaction or individual:

1. Time between electronic market transaction failures $\tilde{D}(t, r)$

2. Claims value each failure: mean, variance, caps $\tilde{C}(m, v, k)$

3. Net premium rate (paid per time period): premiums, costs (depreciating rate), moral hazard adjustment $\mathrm{P}(\mathrm{t}, \mathrm{z}, \mathrm{q})$

The most important parameters will initially be $p, z$ and $r$; as the insurance firm gains claims experience, changes to $\lambda, \mathrm{m}, \mathrm{v}, \mathrm{w}, \mathrm{k}$ and $\mathrm{d}$ are likely as well.

The stream of fund inflows and outflows over time will be $\{P(s \mid t, z, q)-\tilde{C}(s \mid m, v, k) \times$ $\tilde{D}(t, r)\}_{t}$ and the product design problem can be stated as follows:

$$
\begin{aligned}
& \max \left(N P V_{d}[P(t, z, p, q)-E[\tilde{C}(m, v, k) \times \tilde{D}(t, r)]]\right) \\
& \text { Constraint: } A_{P}(t, z, p, q)-\operatorname{VaR}_{\{t, t+1 \mid w\}}[\tilde{C}(m, v, k) \times E[\tilde{D}(t, r)]] \leq L \quad \forall\{t, t+1\}
\end{aligned}
$$


That is, we want to maximize the expected net present value of the future payments for any individual policy, subject to the constraint that the fund of net premium payments at any future time period $\{t, t+1\}$, will exceed the $100 \mathrm{w} \%$ value at risk $V a R_{\{t, t+}$ ${ }_{1 \mid w\}}(\cdot)$ by at least the fund value $L$.

The calculation of $A_{p}(t, c, p, q)$ derives from a sum of geometric series.

$$
A_{P}(t, z, p, q)=\sum_{s=0}^{t}\left(p-z e^{-q s}\right)(1+d)^{s}=p \frac{(1+d)^{t+1}-1}{d}-z \frac{1-\left[e^{-q}(1+d)\right]^{t+1}}{1-e^{-q}(1+d)}
$$

Investors may be interested in imposing additional constraints. For example, that the expected net premium contribution in any period is positive to secure cash flow, i.e., $P(t, z, p, q)-E[\tilde{C}(m, v, k) \times \tilde{D}(t, r)] \geq 0 \quad \forall t$. Or, conversely, that the fund does not exceed a certain value (implying too much cash to manage, or problems in finding assets to invest given the company's risk management targets, i.e., $P(t, z, p, q)-E[\tilde{C}(m, v, k) \times \tilde{D}(t, r)] \leq M$ for some $M$ (Table 3 ).

The constraints imposed here are simpler to impose than the VaR constraint. A VaR constraint is necessary for regulatory approval in insurance firms. Since online auctions avoid such regulations by classifying their transaction insurance as a 'buyer protection program', the net premium constraints are mainly relevant to investors or the owners of the market (e.g., eBay shareholders) and will be reflected in the stock price or cost of capital.

This model assumes that $\tilde{C}$ and $\tilde{D}$ But tools exist to construct $\tilde{C} \times \tilde{D}$ from empirical data about the marginal distributions. The Clayton copula, also referred to as the (Cook and Johnson, 1981) copula, originally studied by (Kimeldorf and Sampson, 1987, 1989) has been used to study correlated insurance and portfolio risks because it exhibits strong left tail dependence and relatively weak right tail dependence. Anecdotal and empirical evidence suggests that loan defaults are highly correlated during recessionary times. Similarly, researchers have studied the "broken heart syndrome" in which spouses' ages at death tend to be correlated. When correlation between two events, such as performance of two funds or spouses' ages at death, is strongest in the left tail of the joint distribution, Clayton is an appropriate modeling choice (Trivedi and Zimmer, 2007). The Clayton kernel takes the form $\mathcal{C}\left(u_{1}, u_{2} \mid \theta\right)=\left(u_{1}{ }^{-\theta}+u_{2}{ }^{-\theta}-1\right)^{-\frac{1}{\theta}} ; \theta \geq 0$. As $\theta$ approaches zero, the marginals become independent. As $\theta$ approaches infinity, the copula attains the FréchetHoeffding upper bound, but for no value does it attain the Fréchet-Hoeffding lower bound. The Clayton copula cannot account for negative dependence. There is little change in this joint distribution for values of $\theta>3$. In both cases, the parameters are set to those used in the rest of this paper's analysis. These $\tilde{C} \times \tilde{D}$ copulas can be estimated from real-world claims experience by sampling fixed periods (e.g., 320 days in the context of the current analysis) and assuming that customer claims behavior is stable over time. It would be feasible as well to use data to infer the value of the coefficient $\theta$ though the attraction of copulas is the reduced information requirements of only having to worry about estimation of the marginal distributions.

The simplest characterization of the transaction failure distribution $\tilde{D}(t, r)$ is exponential and for current purposes, the single parameter exponential distribution provides 
Table 3 Notation used in the Paper

\begin{tabular}{|c|c|}
\hline Parameter & Description \\
\hline$t$ & Time \\
\hline$r$ & Time to the next auction dispute arising from some breach of transaction protocol \\
\hline$m, v$ & Mean and variance of the claims distribution $\tilde{C}(m, v, k)$ \\
\hline k & Cap on claims to control moral hazard in $\tilde{C}(m, v, k)$ \\
\hline$z$ & Operating costs for insurance system \\
\hline$d$ & $\begin{array}{l}\text { The annuity for the net premium fund is computed at } d \text { the same opportunity cost } \\
\text { of capital rate as I use for the NPV calculations. }\end{array}$ \\
\hline$\lambda$ & Lagrange multiplier for optimization \\
\hline 9 & $\begin{array}{l}\text { Cost adjustment depreciation rate (moral hazard declines the longer the insure has } \\
\text { paid into the system through subscriptions) }\end{array}$ \\
\hline$\tilde{C}(m, v, k)$ & Claims: mean, variance, caps on claims payouts \\
\hline$P(t, z, q)$ & $\begin{array}{l}\text { Net premium: premiums, costs (depreciating at q rate), moral hazard adjustment; this } \\
\text { is 'service systems' charge is equivalent to an insurance premium; it is periodic, } \\
\text { non-stochastic, stable over time. For the eBay parameter simulation either, one per } \\
\text { auction listing or one per time period to cover all auctions within that time period } \\
P * \tilde{S}(t) \text { or } P * t \text { For the charts in this section, the premium is set to } 10 \% \text { of the } \\
\text { average sales value of } \$ 85.95 \text { or } \$ 8.60 \text { premium for each sale; this is reasonable } \\
\text { given the anecdotal evidence, but would require further investigation before } \\
\text { finalizing pricing on an actual product }\end{array}$ \\
\hline \multirow[t]{2}{*}{$\tilde{S}(t \mid v)$} & $\begin{array}{l}\text { Random variable of count of sales at time } t \text { where } \lambda \text { is the rate of sales. One listing } \\
\text { every } 6.36 \text { days; } t \text { in days; } v=0.1572 * 57.53 \%=0.09046 \text { sales per day }\end{array}$ \\
\hline & $P[$ there are $k$ sales in period of length $t]=P[\tilde{S}(t \mid v)=k]=\frac{e^{-v t}(v t)^{k}}{k !}$ \\
\hline$\tilde{C}(x \mid a, \beta)$ & $\begin{array}{l}\text { Random variable of value of individual claim. Lomax or Normal (Gaussian). Average } \\
\text { sale is } \$ 85.95 \text { in 2013; and this is assumed to be the average claim (claim for full } \\
\text { sales amount which is typical for auction insurance) }\end{array}$ \\
\hline$\tilde{D}(k \mid t, r)$ & $\begin{array}{l}\text { Time between claims computed as every } \varpi^{\text {th }} \text { sale; we assume every } 221^{\text {st }} \text { sale; } \\
\text { this is reasonable given the anecdotal evidence, but would require further } \\
\text { investigation before finalizing pricing on an actual product }\end{array}$ \\
\hline$\tilde{A}(t \mid P, r, \tilde{S}, \tilde{V}, \tilde{C}, K)$ & Annuity fund $N P V_{t}(\tilde{S}(t) \times P-\tilde{D}(t) \times \tilde{C})$ \\
\hline$\varpi$ & Rate of auction failure as a proportion of auction listings \\
\hline y & Quantile (parameter for the $\mathrm{VaR}$ and tVaR metrics) \\
\hline $\operatorname{VaR}_{y}(\tilde{X})$ & $\begin{array}{l}\text { Value at risk for } y \text { quantile; this is the quantile of the random variable } \tilde{X} \text {. VaR is not } \\
\text { a coherent risk measure, because it violates the subadditivity property - i.e., it } \\
\text { violates } \operatorname{Va} R(X+Y) \leq \operatorname{VaR}(X)+\operatorname{VaR}(Y)\end{array}$ \\
\hline $\operatorname{tVa} R_{y}(\tilde{X})$ & Tail value at risk for $\gamma$ quantile; $t V a R$ is a coherent risk measure \\
\hline
\end{tabular}

an adequate characterization of Internet transaction failure over time. The one period claims random variable $\tilde{C}(m, v, k)$ has a distribution right-censored by the payout cap:

$$
\tilde{C}(m, v, k)=\left\{\begin{array}{cc}
\tilde{C}(m, v) ; & \tilde{C}<k \\
k ; & \tilde{C} \geq k
\end{array}\right.
$$

More specifically, we can use the actuarial statistical notation $\tilde{Z} \wedge k$ to describe a random variable whose behavior is depicted by c.d.f $\tilde{C}(m, v, k)=\left\{\begin{array}{cc}\tilde{C}(m, v) ; & \tilde{C}<k \\ k ; & \tilde{C} \geq k\end{array}\right.$. I will investigate two feasible distributions $F \tilde{C}(x)$ for $\tilde{C}(m, v)$ : the Normal distribution and the Pareto type II (Lomax) distribution. The former is commonly encountered in analyses of financial products, but the latter is common in insurance to model payout distributions, 
because of its positive support and empirical support for its power law distribution of wealth transfers. Its expectation is:

$$
\mathrm{E}[\tilde{C}(m, v, k)]=\int_{-\infty}^{k} x f \tilde{c}(x) d x-k(1-F \tilde{c}(k))=\int_{0}^{k}[1-F \tilde{c}(x)] d x-\int_{-\infty}^{0} F \tilde{c}(x) d x=b_{\max }
$$

Where $f \tilde{c}(\cdot)$ and $F \tilde{c}(\cdot)$ are the pdf and cdf respectively (p.26).

The expectation of $E[\tilde{C}(m, v, k) \times \tilde{D}(t, r)]$ is the product of the stochastically independent severity and frequency random variables $\{\tilde{C}(\cdot), \tilde{D}(\cdot)\}$ and during the one period time interval $\{t, t+1\}$ is:

$$
E[\tilde{C}(m, v, k) \times \tilde{D}(t, r)]=\int_{t}^{t+1} f_{\tilde{D}} d t \times E[\tilde{C}(m, v, k)]=b_{\max }\left[e^{-r t}-e^{-r(t+1)}\right]
$$

Since $f_{\tilde{D}}(t)=1-e^{-r t}$ and thus $\int_{t}^{t+1} f_{\tilde{D}} d t=e^{-r t}-e^{-r(t+1)}$

Here (in terms of a simplified notation) you can use the iterated-expectation method for computing the mean of an expression $g(A, B)$ when $\mathrm{A}$ and $\mathrm{B}$ are themselves random:

$$
E[g(A, B)]=E[E[g(A, B) \mid B]]
$$

where the inner expected value on the right of the equals sign is with respect to $\mathrm{A}$, given $\mathrm{B}$, and the outer expected value is with respect to $\mathrm{B}$. The variance is more complicated:

$$
\operatorname{Var}[\mathrm{g}(\mathrm{A}, \mathrm{B})]=\mathrm{E}[\operatorname{Var}[\mathrm{g}(\mathrm{A}, \mathrm{B}) \mid \mathrm{B}]]+\operatorname{Var}[\mathrm{E}[\mathrm{g}(\mathrm{A}, \mathrm{B}) \mid \mathrm{B}]
$$

where the inner expected value and variance are with respect to $\mathrm{A}$, given $\mathrm{B}$, and the outer expected value and variance are with respect to $B$.

The terms in the NPV calculation are:

$$
P(t, z, p, q)-E[\tilde{C}(m, v, k) \times \tilde{D}(t, r)]=p-z e^{-q t}-b_{\max }\left[e^{-r t}-e^{-r(t+1)}\right]
$$

Substituting and consolidating, the constraint in a given time period is

$$
p \frac{(1+d)^{t+1}-1}{d}-z \frac{1-\left[e^{-q}(1+d)\right]^{t+1}}{1-e^{-q}(1+d)}-\left[e^{-r t}-e^{-r(t+1)}\right] F \tilde{c}^{-1}(w) \leq L \quad \forall\{t, t+1\}
$$

Where $F \tilde{c}^{-1}(w)$ is the quantile function for the distribution of $\tilde{C}$. For the rightcensored random variable, the cdf is 1 at $k$, and thus:

$$
\begin{aligned}
& F_{\tilde{C}(m, v, k)}(x)=\left\{\begin{array}{cc}
\frac{F \tilde{c}(x)}{F \tilde{C}(k)} & x<k \\
1 & x \geq k
\end{array}\right. \\
& F_{\tilde{C}(m, v, k)}^{-1}(w)=F \tilde{c}^{-1}(w) F \tilde{c}(k)
\end{aligned}
$$

Consolidating and rewriting the original problem:

$$
\begin{aligned}
& \max \left(N P V_{d}\left[p-z e^{-q t}-b_{\max }\left[e^{-r t}-e^{-r(t+1)}\right]\right]\right)= \\
& =\max \left(\sum_{t=0}^{\infty}\left[\frac{p-z e^{-q t}-b_{\max }\left[e^{-r t}-e^{-r(t+1)}\right]}{(1+d)^{t}}\right]\right)
\end{aligned}
$$




$$
\cong \max \left(\int_{0}^{\infty}\left[\frac{p-z e^{-q t}-b_{\max }\left[e^{-r t}-e^{-r(t+1)}\right]}{(1+d)^{t}}\right] d t\right)=\max (G(\cdot))
$$

\section{Constraint:}

$p \frac{(1+d)^{t+1}-1}{d}-z \frac{1-\left[e^{-q}(1+d)\right]^{t+1}}{1-e^{-q}(1+d)}-\left[e^{-r t}-e^{-r(t+1)}\right] F_{\tilde{C}(m, v, k)}^{-1}(w)=H(\cdot) \leq L \quad \forall\{t, t+1\}$

Where neither $b_{\max }=\left(\int_{0}^{k}[1-F \tilde{c}(x)] d x-\int_{-\infty}^{0} F \tilde{c}(x) d x\right)$ nor $F_{\tilde{C}(m, v, k)}^{-1}(w)$ contain $t$ and $G(\cdot)$ and $H(\cdot)$ encapsulate their respective expressions. Evaluate $G(\cdot)$

$$
\max \left(p \frac{1}{1-t}(1+d)^{1-t}-\left.\frac{1}{1+t}\left[z\left(\frac{e^{-q}}{1+d}\right)^{1+t}-b_{\max }\left[1-e^{-r}\right]\left(\frac{e^{-r}}{1+d}\right)^{1+t}\right]\right|_{0} ^{\infty}\right)
$$

Since $\lim _{n \rightarrow \infty} \frac{x^{n}}{n}=0$ the integrals under maximization evaluate to 0 at the $\infty$ limit, and the objective function becomes

$$
\max \left(p(1+d)-z \frac{e^{-q}}{1+d}-b_{\max }\left[e^{-r}-1\right] \frac{e^{-r}}{1+d}\right)=\max (G(\cdot))
$$

A sufficient condition for the constraint to hold for each time period is that its minimum must hold. The minimum is found at time $t^{*} \mid \frac{\partial \mathrm{H}(\cdot)}{\partial \mathrm{t}}=0 ; \frac{\partial^{2} \mathrm{H}(\cdot)}{\partial \mathrm{t}^{2}}>0$ and the constraint holds where $\mathrm{H}\left(\mathrm{t}^{\prime \prime},\right) \geq 0$. The first order condition to solve for $t^{*}$ is

$$
\begin{aligned}
& \frac{\partial\left[p \frac{(1+d)^{t+1}-1}{d}-z \frac{1-\left[e^{-q}(1+d)\right]^{t+1}}{1-e^{-q}(1+d)}-\left[e^{-r t}-e^{-r(t+1)}\right] F_{\tilde{C}(m, v, k)}^{-1}(w)-L\right]}{\partial \mathrm{t}}= \\
& =p \frac{(1+t)(1+d)^{t}}{d}-z \frac{(1+t)\left[e^{-q}(1+d)\right]^{t}}{1-e^{-q}(1+d)}-r F_{\tilde{C}(m, v, k)}^{-1}(w)\left[e^{-r t}-e^{-r(t+1)}\right]=0 \\
& \Rightarrow \frac{\left[e^{-r} /(1+d)\right]^{t}}{(1+t)}=\frac{p / d-z \frac{e^{-q}}{1-e^{-q}(1+d)}}{r F_{\tilde{C}(m, v, k)}^{-1}(w)\left[1-e^{-r}\right]}
\end{aligned}
$$

By definition $\mathrm{d}, \mathrm{q}, \mathrm{r}, \mathrm{z}$ and $b_{c o n}$ are positive real numbers, so the second order condition

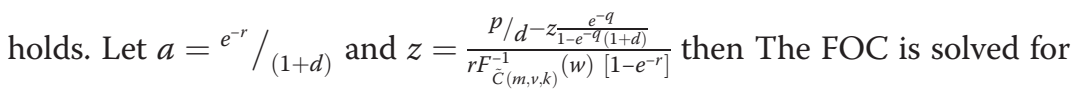

$$
t^{*}=-\left(1+\frac{W\left(-\frac{\ln (a)}{a z}\right)}{\ln (a)}\right)
$$

ere $W(\cdot)$ is the Lambert $\mathrm{W}$-function, implying that $t^{*}$ is either complex or negative, both of which imply that there is no minimum on a positive real support. This in turn implies that if the constraint holds at $t=0$ it will hold for other values.

A necessary condition for a constrained maximum (and also a sufficient one assuming that $G(\cdot)$ is bounded with a single optimum) is provided by the method of Lagrange multipliers, and the Karush-Kuhn-Tucker conditions. Define the Lagrangian $\Lambda(\cdot)$ and Lagrange multiplier $\lambda$; let

$$
\Lambda(p, z, k, q, r, m, v, d, L)=G(\cdot)-\lambda H\left(t^{*}, \cdot\right)
$$


Where $t^{*}$ is a constant in the $\lambda H\left(t^{*}\right.$, ) part of the Lagrangian. The Lagrangian has solution

$$
\begin{aligned}
& \nabla_{p, z, k, q, r, m, v, d, L, \lambda}[\Lambda(\cdot)]=\left[\frac{\partial \Lambda(\cdot)}{\partial \mathrm{p}}, \frac{\partial \Lambda(\cdot)}{\partial \mathrm{z}}, \ldots\right]=0 \\
& \begin{aligned}
\Lambda(\cdot)= & -p(1+d) \\
& +z \frac{e^{-q}}{1+d}-b_{\max }\left[1-e^{-r}\right] \frac{e^{-r}}{1+d}-\lambda\left[p-z e^{-q t^{*}}-b_{c o n}\left[e^{-r t^{*}}\left(1-e^{-r}\right)\right]-L\right]
\end{aligned}
\end{aligned}
$$

Each of the components of the Lagrangian gradient $\nabla .(\Lambda(\cdot))$ is calculated independently below.

Premiums:

$$
\frac{\partial \Lambda(\cdot)}{\partial \mathrm{p}}=-(1+d+\lambda)=0 \Rightarrow \lambda=-1-d ; d=-1-\lambda
$$

i.e., optimization on notional premium $p$ is meaningless since you can always increase NPV by raising $p$. This is consistent with the product being viable assuming the initial premium is adequate to cover costs.

Costs:

$$
\frac{\partial \Lambda(\cdot)}{\partial \mathrm{z}}=\left(\frac{e^{-q}}{1+d}-\lambda \frac{e^{-q t^{*}}}{1+d}\right)=0 \Rightarrow \lambda=\quad e^{t^{*}} ; d, q=\infty \text { and } \frac{\partial \Lambda(\cdot)}{\partial \mathrm{q}}=-\mathrm{z}\left(\frac{e^{-q}}{1+d}-\lambda \frac{e^{-q^{*}}}{1+d}\right) \Rightarrow \lambda=
$$

$\frac{e^{t^{*}}}{z} ; d, q=\infty$ i.e., optimization on SG\&A cost $c$ implies that dropping SG\&A costs to zero, and discounting all future periods (where claims might be possible) is optimal. Optimization on declining balance rate for SG\&A $q$ are not meaningful - you can always increase NPV by lowering costs. This is consistent with the product being viable assuming the first period premium is adequate to cover costs.

\section{Claims distribution}

$$
\begin{aligned}
& \frac{\partial \Lambda(\cdot)}{\partial \mathrm{m}}=-\left(\frac{\partial b_{\max }}{\partial m}\left[1-e^{-r}\right] \frac{e^{-r}}{1+d}-\lambda \frac{\partial b_{\text {con }}}{\partial m}\left[e^{-r t^{*}}\left(1-e^{-r}\right)\right]\right) \\
& \frac{\partial \Lambda(\cdot)}{\partial \mathrm{v}}=-\left(\frac{\partial b_{\max }}{\partial v}\left[1-e^{-r}\right] \frac{e^{-r}}{1+d}-\lambda \frac{\partial b_{c o n}}{\partial \nu}\left[e^{-r t^{*}}\left(1-e^{-r}\right)\right]\right) \\
& \frac{\partial \Lambda(\cdot)}{\partial \mathrm{k}}=-\left(\frac{\partial b_{\max }}{\partial k}\left[1-e^{-r}\right] \frac{e^{-r}}{1+d}-\lambda \frac{\partial b_{c o n}}{\partial k}\left[e^{-r t^{*}}\left(1-e^{-r}\right)\right]\right)
\end{aligned}
$$

i.e., optimization for $m, v, k$ involve the payout distribution $F \tilde{C}(x)$ where derivatives for $b_{\max }$ and $b_{c o n}$ are complicated and are explored later in this paper using numerical methods.

\section{NPV Discounting}

$$
\frac{\partial \Lambda(\cdot)}{\partial \mathrm{d}}=\frac{\partial \Lambda(\cdot)}{\partial(1+\mathrm{d})} \frac{\partial(1+\mathrm{d})}{\partial \mathrm{d}}=\frac{\partial \Lambda(\cdot)}{\partial(1+\mathrm{d})}=-p-z \frac{e^{-q}}{(1+d)^{2}}+b_{\max }\left[1-e^{-r}\right] \frac{e^{-r}}{(1+d)^{2}}
$$

As expected, at the margin, the discount rate has declining influence, since $(1+d)^{2}>$ $(1+d)>1$. 


\section{Auction transaction failures}

$$
\begin{aligned}
\frac{\partial \Lambda(\cdot)}{\partial \mathrm{r}} & =\left(b_{\max } \frac{e^{-r}-2 e^{-2 r}}{1+d}-\lambda b_{\operatorname{con}}\left[t^{*} e^{-r t^{*}}-\left(t^{*}+1\right) e^{-r\left(t^{*}+1\right)}\right]\right)=0 \Rightarrow \lambda \\
& =\frac{b_{\max } \frac{e^{-r}-2 e^{-2 r}}{1+d}}{b_{\text {con }}\left[t^{*} e^{-r t^{*}}-\left(t^{*}+1\right) e^{-r\left(t^{*}+1\right)}\right]} ; d, r=\infty
\end{aligned}
$$

As expected, mean time to failure is $r$ for the exponential distribution, and optimization on $r$ implies that discounting of all future periods, and reducing the Internet transaction failures to zero are optimal. The shadow price $\frac{\partial \Lambda(\cdot)}{\partial \mathrm{L}}=\lambda$, is the marginal change in NPV arising from a change in the reinsurance fund requirement $\mathrm{L}$.

\section{Competing interests}

The author declares that he has no competing interest.

Received: 12 May 2015 Accepted: 12 May 2015

Published online: 09 June 2015

\section{References}

Anythingresearch.com (2013) 2013 market research report on online (internet) auctions

Artzner P, Delbaen F, Eber J-M, Heath D (1999) Coherent measures of risk. Math Finance 9(3):203-228

Basak S, Shapiro A (2001) Value-at-risk-based risk management: optimal policies and asset prices. Rev Financial Stud 14(2):371-405

Bassett GW, Koenker R, Kordas G (2004) Pessimistic portfolio allocation and Choquet expected utility. J Financial Econometr 2(4):477-492

Boyle PP, Hardy M, Vorst TCF (2005) Life after VaR. J Derivat 13(1):48-55

Cook RD \& Johnson ME (1981). A family of distributions for modelling non-elliptically symmetric multivariate data. J Royal Statistical Society. Series B (Methodological), (1981): 210-218.

Daníelsson J, Embrechts P, Goodhart C, Keating C, Muennich F, Renault O, Shin HS (2001) An academic response to basel II: school of economics, financial markets group

Duffie D, Pan J (1997) An overview of value at risk. J Derivat 4(3):7-49

Duffie D, Pan J (2001) Analytical value-at-risk with jumps and credit risk. Finance Stochastics 5(2):155-180

Fermanian J-D, Scaillet O (2005) Sensitivity analysis of VaR and expected shortfall for portfolios under netting agreements. J Bank Finance 29(4):927-958

Gourieroux C, Jasiak J (2010) Value-at-risk, Handbook of financial econometrics., pp 553-609

John MB, Hancock GH (2005) Variance of the CTE estimator. North Am Actuar J 9(2):129-156

Kimeldorf G, Sampson AR (1987) Positive dependence orderings. Ann Inst Stat Math 39(1):113-128

Kimeldorf G, Sampson AR (1989) A framework for positive dependence. Ann Inst Stat Math 41(1):31-45

Lee, Ho Geun, Theodore Clark, and Kar Yan Tam. "Research report. Can EDI benefit adopters?." Information Systems Research 10.2 (1999): 186-195

Lee HG, Lee SC, Kim HY, Lee RH (2003) Is the internet making retail transactions more efficient? comparison of online and offline CD retail markets. Elect Commerce Res Appl 2(3):266-277

Moscadelli M (2004) The modelling of operational risk: experience with the analysis of the data collected by the Basel Committee. Available at SSRN 557214

Rockafellar RT, Uryasev S (2000) Optimization of conditional value-at-risk. J Risk 2:21-42

Rockafellar RT, Uryasev S (2002) Conditional value-at-risk for general loss distributions. J Bank Finance 26(7):1443-1471

Sharpe WF, Sharpe WF (1970) Portfolio theory and capital markets (Vol. 217). McGraw-Hill, New York

Tasche D (2002) Expected shortfall and beyond. J Bank Finance 26(7):1519-1533

Trivedi PK, and Zimmer DM. Copula modeling: an introduction for practitioners. Now Publishers Inc, 2007

Wolpert RL (2012) Extremes. 2013, from http://www.stat.duke.edu/courses/Fall09/sta104.02/lec/104wk05.pdf

\section{Submit your manuscript to a SpringerOpen ${ }^{\circ}$ journal and benefit from:}

- Convenient online submission

- Rigorous peer review

- Immediate publication on acceptance

- Open access: articles freely available online

- High visibility within the field

- Retaining the copyright to your article 